\section{Cahiers de Narratologie}

Analyse et théorie narratives

$10.1 \mid 2001$

La voix narrative

\title{
Ambivalence de la voix narrative et effets d'ironie : Bartleby (H. Melville) et Un petit bout de femme (F. Kafka)
}

Florence Godeau

\section{CpenEdition}

Journals

Electronic version

URL: http://journals.openedition.org/narratologie/6983

DOI: 10.4000/narratologie.6983

ISSN: 1765-307X

Publisher

LIRCES

\section{Printed version}

Date of publication: 1 January 2001

Number of pages: $503-511$

ISBN: 2914561032

ISSN: 0993-8516

Electronic reference

Florence Godeau, "Ambivalence de la voix narrative et effets d'ironie : Bartleby (H. Melville) et Un petit bout de femme (F. Kafka)", Cahiers de Narratologie [Online], 10.1 | 2001, Online since 20 November 2014, connection on 23 February 2021. URL: http://journals.openedition.org/narratologie/6983 ; DOI: https:// doi.org/10.4000/narratologie.6983 


\title{
AMBIVALENCE DE LA VOIX NARRATIVE ET EFFETS D'IRONIE :
} « BARTLEBY » (H. MELVILLE) ET « UN PETIT BOUT DE FEMME » $(\text { F. KAFKA })^{1}$

\author{
Florence GODEAU \\ Université de Versailles
}

Entre "Bartleby, the Scrivener », publié en 1853, et "Eine kleine Frau », publié en 1924, existent de frappantes affinités thématiques et formelles, en dehors de toute influence directe ou indirecte. Ces deux récits, où un narrateur autodiégétique anonyme détient l'exclusivité du point de vue sur les faits, évoquent une obsession confinant à la paranoïa. Dans la nouvelle de Melville, un être $a$ priori insignifiant pousse à bout son employeur par sa résistance passive apparemment immotivée, tandis qu'un "petit bout de femme " de rien du tout semble se complaire, pour des raisons obscures, à tourmenter le narrateur kafkaien. Dans l'un et l'autre textes, le mode et la voix pseudo-autobiographiques nous invitent, en première analyse, à partager les désarrois du "je". Cependant un certain nombre d'indices signalent une " mise en scène ironique », selon la terminologie de Philippe Hamon², frappant d'un soupçon la teneur et la forme sérieuses du discours, et exigeant une lecture seconde. L'auteur ${ }^{3}$, ironi-

1 Éditions utilisées: F. Kafka, "Eine kleine Frau", dans Die Erzählungen, Fischer, Frankfurt am Main, 1998 (abrégé comme suit : " $K F$ " suivi du numéro de page); "Un petit bout de femme", dans $U n$ jeûneur et autres nouvelles, trad. B. Lortholary, Paris, GF, 1993 (abrégé comme suit : "PBF" suivi du numéro de page) ; H. Melville, "Bartleby", Penguin Books, 1995 (abrégé comme suit : "B", suivi du numéro de page); trad. M. Causse, dans Bartleby. Les Iles enchantées. Le campanile, Paris, GF, 1989 (abrégé comme suit : "B" suivi du numéro de page).

2 P. HAMON, L'Ironie littéraire, Paris, Hachette, 1996.

3 L'emploi de ce terme paraît autorisé par la "théorie intersubjective" du récit romanesque exposée par Maurice Couturier ( $\mathrm{La}$ 
sant en coulisses, sollicite implicitement notre complicité face à un narrateur personnel dont nous ne saurions être tout à fait dupes, même s'il se livre à un plaidoyer pro domo. Son antagoniste, qui plus est, n'a guère voix au chapitre : si la formule bartlebyenne I would prefer not to et ses variantes nous sont "rapportées" par le narrateur melvillien, nous n'entendrons jamais la "petite femme" du récit de Kafka, sans voix et sans nom, mais dont la gestuelle s'avère en revanche particulièrement expressive... A travers une lecture à "double foyer", en quelque sorte, nous voudrions montrer que l'ambivalence de la voix narrative est ici la source d'une tension féconde entre sympathie et distanciation, caractéristique de l'ironie « moderne », selon Philippe Hamon.

"Objet" de l'histoire, "sujet" de l'énonciation : une relation ambivalente

"Bartleby" et "Un petit bout de femme" semblent fondés sur une ellipse du sens, au niveau diégétique, doublée d'un jeu complexe sur l'absence, voire la rétention d'informations, au niveau de la narration.

Le "mystère Bartleby" pourrait demeurer aussi opaque que ce dead-wall devant lequel l'employé semble... dormir debout. Dès l'incipit, derrière les figures de rhétorique et autres hyperboles soulignant le caractère extraordinaire de l'objet du récit, affleure l'incapacité à dire quoi que ce soit de "sûr" concernant Bartleby. La pénurie est à la source du texte. Ce "témoignage" supposé ne pourra être que l'expression

figure de l'auteur, Paris, Seuil, 1995), ainsi que par les mises au point proposées par Vincent Jouve dans son ouvrage La lecture (Paris, Hachette, 1993) ainsi que dans le cadre de ce colloque (v. infra). La relation auteur/lecteur/narrateur-personnage est au cœur de toute analyse des effets de lecture, auxquels l'ironie doit évidemment être rattachée.

4 Par exemple, lorsque le "je" kafkaïen déclare avoir voulu un jour suggérer à son antagoniste "la meilleure façon de mettre un terme à cette irritation" (KF, 509; PBF, 62), il se garde de nous dire en quoi consistait ce conseil qui "n'a servi qu'à provoquer un tel emportement que jamais plus [il] ne renouveller[a] cette tentative." (ibid.) Le narrateur melvillien quant à lui ne précise pas la teneur des "quelques mots" échangés avec Bartleby lors de leur première rencontre (B., 9; B., 18). 
d'une subjectivité ayant un jour découvert l'Inconnu, non seulement dans le monde sur lequel elle prétendait légiférer, mais surtout en elle-même. Confronté à cette page blanche qu'est Bartleby, l'homme de loi, dépouillé de toute omniscience, écrira sous la dictée de l'imaginaire, entreprise à coup sûr périlleuse pour celui qui se trouve a priori le moins bien désigné pour cela... Tout au long du récit, à l'occasion de ses "dialogues" embryonnaires avec l'unaccountable scrivener (B., 20 ; B., 30), ou bien en son for intérieur, le laywer débat et se débat, d'hypothèses en réfutations. L'épilogue, fondé sur des on-dit, et l'exclamation finale ("Ah, Bartleby! Ah, humanité !"), bien loin de conclure, semblent inviter le lecteur, une fois le livre refermé, à l'infini de la méditation. Le sens ultime d'"Un petit bout de femme" lui aussi ne cesse de fuir, si l'on ose cette métaphore deleuzienne, hors de ce tonneau des Danaïdes qu'est le discours du "je". D'une densité presque étouffante, dont la structuration maniaque relève de la pure rhétorique, ce réseau de conjectures tourne à vide, telle une machine célibataire. Chaque assertion, sitôt énoncée, se voit contredite, chaque hypothèse demeure suspendue dans l'indécidable.

L'objet apparent du discours, désigné par le titre, incarne donc chez Melville comme chez Kafka une altérité inconnaissable qui n'en constitue pas moins la seule raison d'être du récit, et, partant, du narrateur. Le zèle des protagonistes, soucieux de convaincre, s'avère cependant handicapé par leurs affects, propres à gauchir toute analyse, mais qui donnent aussi aux voix narratives leur coloration et leur timbre spécifiques. Dans l'un et l'autre textes, le je-narrateur invite son interlocuteur virtuel non seulement à se poser les mêmes questions que lui, avec la même curiosité agitée, mais à partager les mêmes sentiments ambigus à l'encontre de l'unique objet de son ressentiment... Face à cette autorité discursive omniprésente et pressante, soit le lecteur emprunte les mêmes voies interprétatives, et aboutit aux mêmes apories, soit il s'en dissocie, pour poser au texte (dont le narrateurpersonnage n'est qu'une composante spécifique) d'autres questions.

Les douteuses prérogatives du narrateur personnel 
De fait, l'ensemble des signaux attestant chez Melville la prise de distance de l'instance auctoriale par rapport au personnage du lawyer, et, chez Kafka, les effets d'autopastiche ${ }^{5}$ ainsi que les indices signalant une intention ironique sollicitent une analyse sceptique de ces voix narratives tyranniques qui, en parlant de l'"autre", se révèlent autres que ce qu'elles veulent bien paraître...

L'inquiétude du lecteur (inquiétude au second degré, qui redouble et domine celle que disent éprouver les narrateurs de nos deux récits) est éveillée au premier chef par la surabondance des indices d'attestation des faits, contredite par la présence non moins importante des figures du doute... Dans "Bartleby", le jeu des oxymores, les nombreuses formulations binaires, le balancement syntaxique entre hypothèses et rétractations subséquentes soulignent l'ambiguïté de tout ce qui a trait au scribe, l'invalidité de tout jugement définitif à son encontre : à cette écriture de l'incertitude, reflétant la vision du monde de Melville, s'opposent les affirmations péremptoires destinées à donner à la lecture moralisante et tendancieuse du je-narrateur "interprétant" les faits et gestes du copiste l'apparence de la vérité... On notera en particulier l'usage spécieux du présent de vérité générale dans l'argumentaire du lawyer, dès lors qu'il cherche à plaider sa propre cause et à substituer aux doutes qui l'assaillent les préceptes et préjugés de la société où il est lui-même, volens nolens, à la fois juge et partie.

Kafka pour sa part non seulement exploite l'ambiguïté du lexique ${ }^{6}$ mais pousse à l'extrême une rhétorique du para-

5 Cette nouvelle, à bien des égards, peut apparaître comme une variante singulièrement condensée du Procès, rédigé dix ans auparavant.

6 En jouant, à son habitude, sur les connotations d'un même terme ou sur les effets d'écho, comme dans ce passage, dont la traduction ne peut tout à fait restituer la bizarrerie drolatique : "wenn man das Leben in allerkleinste Teile teilen und jedes Teilchen gesondert beurteilen könnte, wäre gewiß jedes Teilchen meines Lebens für sie ein Ärgernis." (KF, 508; PBF, 61-62) ; l'écrivain, par delà son narrateur, qui paraît radoter et marmonner dans sa barbe, joue sur l'accumulation en une même phrase deTeil (morceau), Teilchen (petit morceau, renvoyant du même coup au "petit bout" de femme), teilen (couper, séparer), beurteilen (juger = cf. Das Urteil, "Le verdict"). 
doxe où non-A est toujours aussi vraisemblable que $\mathrm{A}$, tandis que le sujet de l'énonciation lui-même se dérobe constamment à l'analyse, du fait de son essentielle ambivalence : il se prétend au-dessus de tout soupçon, affirme être seul à connaître les tenants et les aboutissants de l'"affaire", ou, pour mieux dire, de cette chose impure (diese unreine Sache) dont on ne saurait parler qu'à mots couverts, par ellipses, allusions et autres prudes et prudentes précautions, fort inutiles en vérité, et fort ambiguës, par surcroît, de sorte qu'à leur tour l'impureté les menace... Mais les assertions péremptoires sont bientôt remises en cause, les certitudes présumées s'opposent aux multiples interrogations, tours hypothétiques et autres manifestations stylistiques du doute et de l'hésitation. A l'instar de Goliadkine, le Double de Dostoïevski, le "je" kafkaïen est ceci et cela, à la fois présomptueux et terriblement peu sûr de lui, retors et naïf, sincère et de mauvaise foi. Par surcroît, ses propos sont susceptibles d'une lecture seconde, que lui-même ne paraît pas soupçonner... Lorsqu'il attribue à son ennemie telle pensée insidieuse dont il serait victime, il décrit en fait son propre mode de fonctionnement, et forge sans le savoir une accusation susceptible de se retourner contre lui : "Je soupçonne même qu'elle ne fait - en partie tout au moins - que simuler la souffrance afin de faire porter sur moi les soupçons du monde." (KF, 510 ; PBF, 63)

Cette réversibilité-réflexivité du discours peut prendre la forme de la contradiction interne la plus explicite : le narrateur se juge digne de confiance, et plein de confiance (Vertrauen verdienend faisant écho à vertrauensvoll) ${ }^{7}$, alors que tout son discours dément cette assurance rassurante et cette bonne réputation supposée. Autopersuasion ?8 Mauvaise foi, consciente ou inconsciente ? Il est malaisé d'en décider : ces deux interprétations sont rendues plausibles par une écriture si savamment équilibrée que jamais la balance de notre propre jugement ne peut pencher de façon décisive d'un côté ou de l'autre... Tout juste pouvons-nous deviner en cette "petite femme" hypernerveuse, soupçonneuse, antipathique, hypo-

$7 \mathrm{KF}, 516$; PBF, 69.

8 Ainsi l'animal du "Terrier" se berce-t-il de paroles rassurantes, auxquelles il ne croit même pas, lorsqu'il se sent menacé par le "bruit" qu'il croit percevoir. 
condriaque et collante ${ }^{9}$, aux dires du narrateur, la part mauvaise du "je" lui-même, l'incarnation toute vive de la haine de soi ; le suicide est du reste brièvement évoqué, fût-ce pour être sitôt après tourné en dérision par un trait d'humour noir particulièrement sarcastique : "ses accès de rage en apprenant, par exemple, mon suicide, ne connaîtraient pas de bornes." (KF, 513 ; PBF, 66)

Le lawyer, quant à lui, se présente comme un homme de confiance ${ }^{10}$ et de bonne foi, "éminemment SÛR" (B., 2 ; B., 10) : un vrai coffre-fort, en quelque sorte, en vertu des deux sens du mot safe, selon qu'il est adjectif ou nom commun... Mais, d'entrée de jeu, le texte de Melville souligne la distorsion entre l'énergique affirmation d'une autorité sociale et discursive sans faille, et l'échec patent de cette autorité même... Sous le glacis d'un autoportrait complaisant et d'un discours lénifiant, transparaît l'inhumanité des rapports hiérarchiques au sein d'une société américaine où les employés se soumettent à la loi de la rentabilité, tandis que leur employeur, qui se pique de charité, use à leur encontre de comparaisons révélatrices : "De même qu'un cheval, indocile, rétif, sent son avoine, de même Dindonneau sentait sa redingote. Elle le rendait insolent. C'était un homme que la prospérité gâtait." (B., 7 ; B., 16)

Cas de conscience fait homme, le copiste angélique et démoniaque échappe pour sa part à l'emprise de la raison raisonnante et de la morale puritaine. Constamment, si l'on ose ainsi parler, le discours du lawyer oscille, dans ses parties "réflexives", entre sincérité et mauvaise foi, et, dans ses parties narratives, entre le souci de rendre compte scrupuleusement des "formules" bartlebyennes en les recopiant à la lettre, et le péché de présomption, la tentation de l'interprétation et de la réécriture des propos et comportements du scribe...11 L'épisode central de la nouvelle est à cet égard révélateur. Un dimanche matin, l'avoué, qui s'apprêtait à se rendre à l'église

9 Elle partage cette kafkaïenne propriété avec les "aides" de K. l'Arpenteur, et les balles de celluloïd de Blumfeld...

10 On sait que pour Melville le "grand escroc" n'est autre que le confidence man...

11 V. Mathieu LINDON, "Description d'un combat", Delta, $\mathrm{n}^{\circ} 6$, avril 1978, pp. 5-27. 
de la Trinité pour y entendre un beau sermon, passe inopinément à son bureau et découvre que Bartleby y a élu domicile. D'abord bouleversé par cette preuve tangible d'une indicible misère, le narrateur finit par attribuer à une folie "incurable et innée" ce qui devrait être mis sur le compte d'une société inique. L'argumentaire est très sophistiqué : après avoir déploré la situation du copiste, en un discours digne d'un prédicateur (exclamatives, recours à la fonction phatique, références à Petra et Carthage), le lawyer justifie son étrange "conversion" en convoquant de manière spécieuse ce qu'il est convenu d'appeler la charité chrétienne :

Pour un être sensible, il n'est pas rare que la pitié soit une souffrance. Et quand il apparaît en définitive que la pitié ne saurait conduire à un secours efficace, le sens commun exige que l'âme s'en débarrasse [common sense bids the soul be rid of it]. (B., 23 ; B., 33)

La cause patente de l'attitude de Bartleby (la misère) est séparée de ses effets logiques (mélancolie, habitudes étrangement économiques), et remplacée par une cause latente purement hypothétique : les "tranquilles mystères" du scribe, précédemment évoqués, sont désormais considérés comme autant de preuves de la "nature morbide de ses humeurs". La peur du jugement social pervertit la vertu innée de l'homme de loi : sa compassion ne pourra plus se manifester qu'à travers cet ultime examen de conscience qu'est le récit luimême.

De fait, à travers l'épilogue, s'accomplit une authentique métamorphose: l'avoué new-yorkais, naguère copie conforme et conformiste de tant d'autres membres de sa caste, est devenu un écrivain original, fasciné par le mystère d'autrui, par sa différence. La voix narrative homodiégétique s'élève en ces dernières pages à l'unisson de celle de l'auteur, dont elle reproduit, positivement cette fois, le style et la vision du monde. L'"original" et la "copie" se confondent en une même parole, vibrant d'une émotion communicative, le "je" atteint à l'universel, au cœur même d'une expression puissamment lyrique.

Le monopole du point de vue et de la voix accordé à un je-narrateur autodiégétique n'exclut pas la présence invisible 
et subtile d'un autre regard, invitant à une autre lecture. Lector sum, ergo dubito. A l'issue de cette brève étude comparée, la topographie ironiste de nos deux nouvelles pourrait être ainsi formulée : dans " Bartleby », le laywer est à la fois le "gardien de la loi" et "l'ironisé", tandis que le narrateur d' "Eine kleine Frau » est à la fois 1'"ironisant" visant la "gardienne de la loi" (qu'il nomme du reste sa "petite juge"), et la cible de l'auto-critique sans complaisance à laquelle se livre Kafka à travers ce double fictionnel. Par ailleurs, dans le texte de Melville, l'évolution du narrateur, lisible dans les dernières pages du récit, manifeste une prise de distance du "je" par rapport à lui-même. Naguère homme de préjugés et de postulats, le lawyer, grâce à Bartleby, a découvert le champ indéfini des possibles, et le doute. Le désir d'écrire l'histoire de l'inénarrable copiste est la preuve et la conséquence de cette révélation. Kafka en revanche entérine l'impossibilité de toute évolution et de toute prise de distance chez son alter ego, dont il tourne en dérision la pusillanimité mortifère, la monomanie célibataire, tout en exprimant à travers lui une vision du monde déchirée et déchirante. La mort elle-même ne saurait "achever" le procès, l'atermoiement perpétuel du verdict. La seule modification possible n'est autre qu'une aggravation présentée comme insensible de l'"inquiétude" du narrateur: la litote spécieuse, l'inutile précaution, révèle et dissimule une paranoïa peut-être justifiée (nous ne le saurons jamais), cristallisée en cette petite personne en apparence insignifiante, et en vérité polysémique et polyvalente, elle aussi bourreau et victime, virile et féminine, juge et partie. Ce possible avatar du "je" (qui "double" ce dernier tout en étant "doublé" par lui, dans la mesure où sa pantomime muette est "commentée" par une voix off) incarne tout ce qui terrifie le narrateur sans qu'il ose (se) l'avouer, c'est-à-dire tout ce que l'auteur a toujours su ne pouvoir jamais supporter, sans jamais hésiter à le dire : la Femme, ou plutôt l'Eternel féminin kafkaïen, avec son cortège de calamités (curiosité excessive, possessivité, dépendance vis-à-vis d'une famille susceptible de demander des comptes); la Société tout entière, importune, fâcheuse, inquisitrice, tyrannique ; tout ce qui, de près ou de loin, compromet la liberté célibataire, le droit de s'en tenir à soi, de n'être qu'à soi. Si le 
texte de Melville ouvre sur l'Infini de l'Imaginaire, en une exclamation par laquelle la voix s'élève, le texte de Kafka nous confine au tréfonds d'une conscience labyrinthique, où se fait entendre, un peu étouffée, haletante, tantôt anxieuse, tantôt mordante, une parole d'outre-monde, la voix blanche de l'écriture, gravée dans les sillons du papier, de la pierre, de la chair. 